\title{
Dapsone-induced erythroderma with Beau's lines
}

\author{
A H PATKI* \& J M MEHTA $\dagger$ \\ * Dr Bandorawalla Leprosy Hospital, Kondhawa, Pune 411022, \\ India; and †Poona District Leprosy Committee, 'Manisha' 2nd \\ floor, Flat No. 35, 2-A, Moledina Road, Pune 411001, India
}

\section{Accepted for publication 27 July 1989}

\begin{abstract}
Summary A 35-year-old female with borderline lepromatous (BL) leprosy who suffered from dapsone-induced erythroderma is reported. Sudden onset of erythroderma gave rise to a temporary arrest of the function of nail matrix with the resultant Beau's lines. She rapidly recovered with omission of dapsone and therapy with systemic corticosteroids and a topical emollient. In view of the potentially fatal hypersensitivity reaction, we suggest that any patient on multidrug therapy for leprosy needs an urgent referral to a dermatologist if the patient developes a skin rash during the first two months of treatment.
\end{abstract}

\section{Introduction}

Diaminodiphenylsulphone (dapsone), the most widely used antileprosy drug, is remarkably nontoxic. In addition to its prolonged and extensive use in the treatment of leprosy, it also has been widely used in some other dermatological conditions like dermatitis herpetiformis, bullous pemphigoid, subcorneal pustular dermatosis, acne conglobata, erythema elevatum diutinum and vesiculobullous lesions of lupus erythematosus. ${ }^{1.2}$ The hypersensitivity reaction to dapsone is usually confined to the first 6 weeks of treatment and may manifest with fever, eosinophilia, mononucleosis, hepatitis, lymphadenopathy and a skin rash. ${ }^{3}$ In the early years of its use, it was termed 'Dapsone syndrome' and was frequent when high doses of dapsone were used. However, it rapidly became infrequent between the mid 1950s and the late 1970s when low doses of the drug were in vogue. ${ }^{4}$ There are sufficient reasons to believe that the hypersensitivity reactions to dapsone are becoming more frequent. ${ }^{4.5}$

We are reporting one case in which erythroderma of sudden onset was the only feature of dapsone hypersensitivity.

\section{Case report}

A 35-year-old female presented to an urban health clinic on 13 September 1988 with erythematous skin lesions on face, back, hands and knees. She had not received any treatment. On examination, she was found to have erythematous infiltrated plaques on face, back, dorsa of hands and knees. 
Both the ulnar, lateral popliteal and posterior tibial nerves were thickened and tender. Her bacteriological index was found to be $2+$ on the Ridley scale. The attending physician diagnosed her as a case of multibacillary leprosy (BL) and put her on multidrug therapy as recommended by the WHO, dapsone ( $100 \mathrm{mg} /$ day $)$, rifampicin $(600 \mathrm{mg} / \mathrm{month}$, supervised) and clofazimine $(300 \mathrm{mg}$ once a month, supervised and $50 \mathrm{mg} /$ day). The first month was uneventful and she was asked to continue the therapy. On 15 October, she noticed scaly, erythematous lesions on face, trunk and extremities. These were present on the skin not previously affected by the leprosy lesions. Thinking that she might be having a 'lepra reaction', she continued to take the prescribed drugs. The rash rapidly generalized with a stinging and burning sensation all over the body. On 1 November she was referred by the attending physician to our hospital as a 'lepra reaction' case.

On admission, she was found to have exfoliative dermatitis. The previous skin lesions of BL leprosy could not be identified due to the diffuse erythema and scaling all over the body (Figure 1). Both the ulnar, lateral popliteal and posterior tibial nerves were thickened and tender. She was afebrile and there was no evidence of icterus, generalized lymphadenopathy or hepatomegaly. Systemic examination revealed no abnormality. Examination of fingernails revealed transverse lines on all the fingernails about $3 \mathrm{~mm}$ anterior to the proximal nail fold (Figure 2). The laboratory investigations revealed the following: haemoglobin $120 \mathrm{~g} / \mathrm{l}$; total leukocyte count $8.6 \times 10 \mathrm{~g} / \mathrm{l}$; polymorphs $65 \%$; lymphocytes $31 \%$; monocytes $2 \%$; and eosinophils $2 \%$. Urine examination did not reveal any abnormality. Bacteriological index was $2+$ (Ridley scale) and morphological index $5 \%$. Serum bilirubin was $11 \mu \mathrm{mol} / \mathrm{l}$, SGPT 7 units $/ \mathrm{l}$ and serum alkaline phosphatase was $46 \mu / \mathrm{l}$.

She was diagnosed as a case of exfoliative dermatitis most probably induced by dapsone.

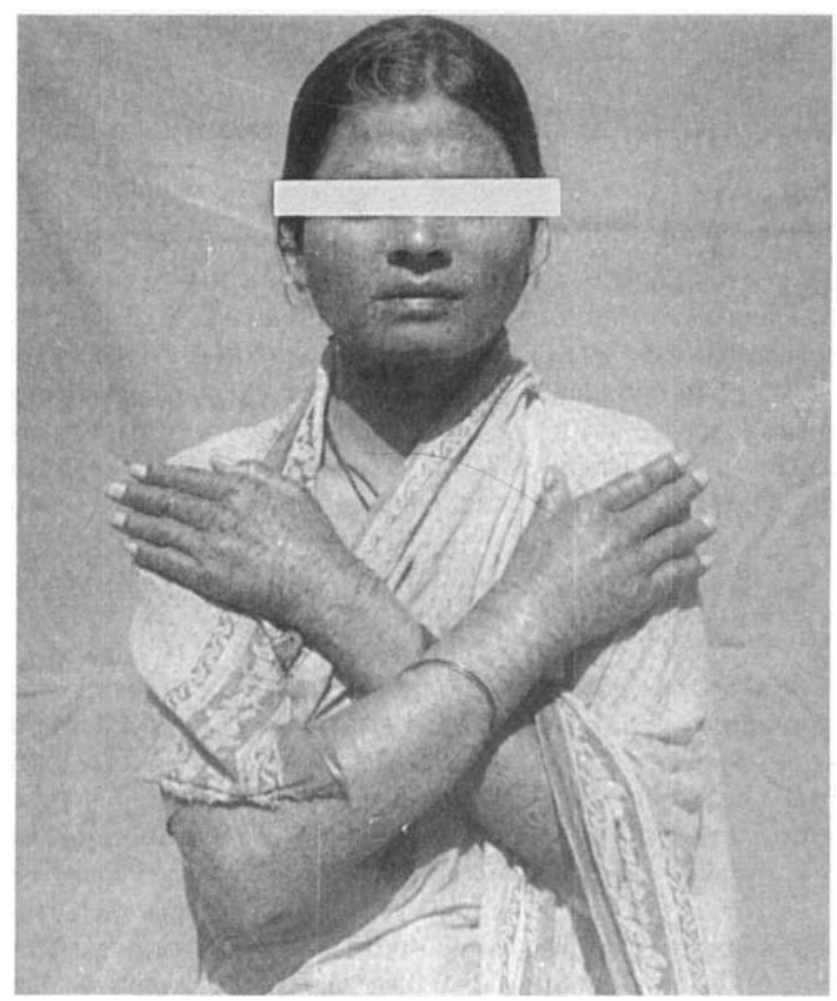

Figure 1. Generalized exfoliative rash. 


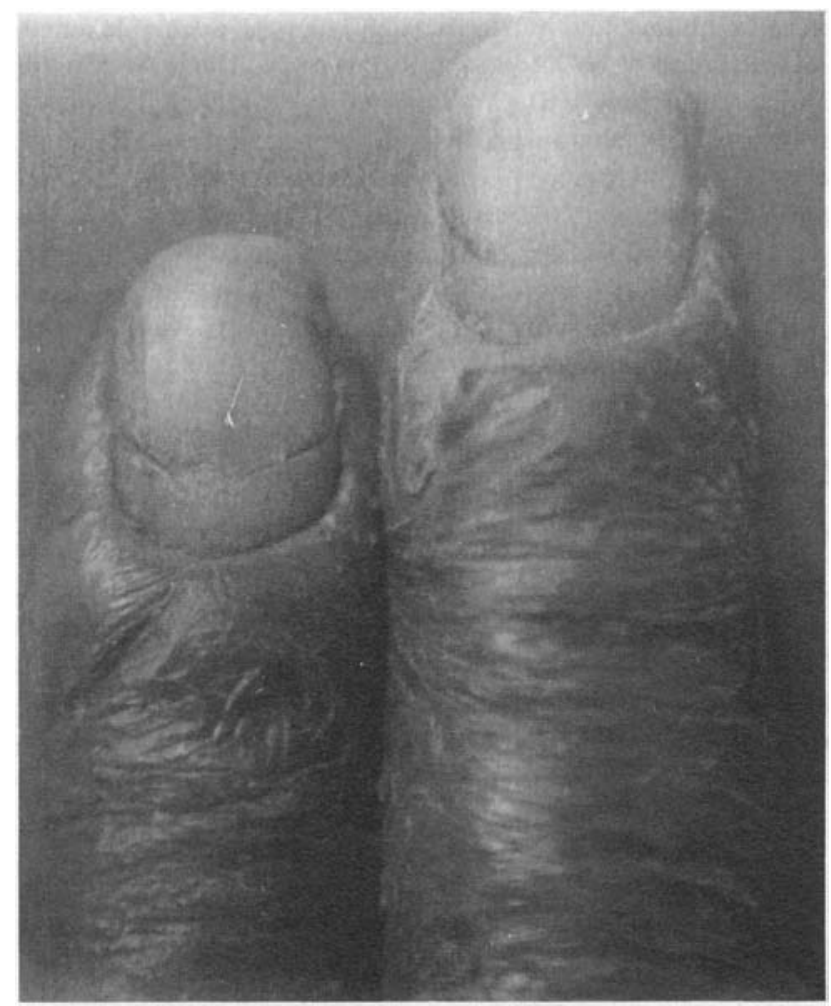

Figure 2. Beau's lines.

Dapsone was omitted and she was put on oral prednisolone $30 \mathrm{mg}$ /day in three divided doses and topical application of petrolatum jelly. Clofazamine and pulsed dose of rifampicin were continued. At the end of one week a marked improvement was seen and the scaling and erythema had reduced. The dose of prednisolone was reduced to $15 \mathrm{mg}$ /day in divided doses. At the end of the second week, the erythema and scaling had completely disappeared and the original leprosy lesions were not visible. Prednisolone was omitted at the end of the second week and she was discharged from the hospital with the advice not to take dapsone again. Isoniazid $300 \mathrm{mg} /$ day was substituted in place of dapsone and she was asked to continue clofazimine and rifampicin.

\section{Comments}

Dapsone hypersensitivity was recognized in $1950^{6}$ and was termed 'Dapsone syndrome' by Allday \& Barnes. ${ }^{7}$ It is characterized by dermatitis, hepatitis, generalized lymphadenopathy and mononucleosis. Of these various features, dermatitis is always present. ${ }^{7}$ Our case was peculiar in that exfoliative dermatitis was the only feature of dapsone hypersensitivity.

Beau's lines are due to a temporary alteration of nail growth rate and occur as a result of measles, mumps, pneumonia, myocardial infarction, pulmonary embolism, zinc deficiency etc. ${ }^{8}$ They are said to be frequent in exfoliative dermatitis. ${ }^{9}$ Although nail matrix arrest occurs in all the erythrodermas, it is of a shorter duration in drug-induced erythrodermas if the offending drug is quickly omitted. This results in resumption of normal nail plate formation. The changes are less dramatic in toe nails because of their slower growth rate and thickness. The depth and width of 
Beau's lines will depend on the severity and duration of the illness. Recently, a sign termed 'shoreline nails' has been described ${ }^{10}$ in patients with recurrent drug induced erythroderma leading to waves of interrupted nail plate formation preceded by a white leukonychial band. The cessation of nail matrix function due to stressful condition is similar to telogen effluvium where the hair follicles in anagen phase are converted to telogen phase.

We attribute the Beau's lines to localized thinning of the nail plate due to the acute onset of erythroderma causing defective nail plate formation. It has been shown that the linear nail growth is not affected during the episode of formation of Beau's lines. ${ }^{11}$ The observation that the lines were 3$\mathrm{mm}$ distal to the proximal nail fold even though the onset of erythroderma was only 15 days ago could be explained by the fact that the nails grow faster in cases of skin disorders with increased epidermal turnover. Examples of these disorders in which increased growth rate of the nails have been reported include psoriasis, ${ }^{12}$ pityriasis rubra pilaris, ${ }^{13}$ and bullous ichthyosiform erythroderma. ${ }^{14}$ In cases of exfoliative dermatitis like the one observed by us, the epidermal turnover is faster ${ }^{15}$ and this may have been responsible for the faster distal movement of the Beau's lines after their formation.

In view of the increasing incidence of dapsone hypersensitivity and potential fatality of the condition, we suggest that any leprosy patient who develops a rash during the first two months of treatment needs an urgent referral to a dermatologist.

Dapsone hypersensitivity has to be differentiated from both Type 1 and Type 2 reactions which may occur in the course of chemotherapy of leprosy. It is differentiated by the rash which causes pruritus and burning and is more or less generalized. In contrast, the lesions of Type 1 reaction consist of erythema and swelling of pre-existing lesions and the appearance of new lesions because inapparent lesions may become clinically apparent. Type 2 reaction gives rise to erythematous, tender and discrete nodules especially on face, arms and thighs.

Dapsone has to be avoided in a patient who develops hypersensitivity. We do not advocate desensitization as suggested by Browne ${ }^{16}$ because of the availability of other antileprosy drugs. Because of the high prevalence of leprosy in India - a total of 4.5 million cases-dapsone is used extensively. Cases of dapsone hypersensitivity are reported in which the syndrome displays several features simultaneously including erythroderma. In the case under report, an incomplete form showing only erythroderma was presented - this being a rare and unique feature. It is also relevant that though the patient continued with dapsone for a period of 15 days after developing erythroderma, no additional signs of dapsone hypersensitivity syndrome appeared.

\section{References}

1 Barranco VP. Dapsone-other indications. Int J Dermatol, 1982; 21: 513-14.

2 Maddin S. Current Dermatologic Therapy. Philadelphia: WB Saunders Company, 1982; 550-1.

3 Jopling WH. Side effects of antileprosy drugs in common use. Lepr Rev, 1983; 54: 261-70.

${ }^{4}$ Smith WCS. Are hypersensitivity reactions to dapsone becoming more frequent? Lepr Rev, 1988; 59: 53-8.

5 Joseph MS. Hypersensitivity reaction to dapsone. Four case reports. Lepr Rev, 1985; 56: 315-20.

${ }^{6}$ Lowe J. Treatment of leprosy with diamino diphenyl sulphone by month. Lancet, 1950; i: 145-50.

7 Allday EJ, Barnes J. Toxic effects of DDS in treatment of leprosy. Lancet, 1951; ii: 205-6.

8 Samman PD, Fenton DA. The nails in disease. London: William Heinemann Medical Books, 4th ed. 1986; $105-7$.

9 Pardo Castello V, Pardo OA. Diseases of the nails. Springfield: Charles C. Thomas Publisher, 3rd ed. 1960; 170.

${ }^{10}$ Shelley WB, Shelley ED. Shoreline nails; sign of drug-induced erythroderma. Cutis, 1985; 35: 220-4.

11 Colver GB, Dawber RPR. Multiple Beau's lines due to dysmenorrhoea. Brit J Dermatol, 1984; 111: 111-13.

12 Dawber R. Fingernail growth in normal and psoriatic subjects. Brit J Dermatol, 1970; 82: 454-7.

13 Dawber R. The ultrastructure and growth of human nails. Arch Dermatol Research, 1986; 269: 197-204.

14 Samman PD. The nails in disease, 3rd; London: William Heinemann Medical Books, 1978; 14.

15 Freedberg IM, Baden HP. Exfoliative dermatitis, In: Dermatology in General Medicine, 3rd ed. Fitzpatrick TB, Eisen AZ, Wolff K et al (eds.), New York: McCraw-Hill Book Company, 1987; 502-5.

16 Browne SG. Desensitization of dapsone dermatitis. Br Med J, 1963; 2: 664-6. 